\title{
Panhypopituitarism in a patient with Thalassemia Intermedia
}

\author{
Chng Chiaw Ling \\ Singapore General Hospital
}

These are the magnetic resonance images (MRI) of the pituitary gland of a 37-year old male with $\beta$-thalassemia intermedia diagnosed at age 17, who was referred to the Endocrine clinic by his hematologist for evaluation of hypogonadism. He was on monthly blood transfusion between 2002 and 2007 and was started on iron chelation in 2003, with hemoglobin maintained between 8-9 g/dL. His condition was complicated by pancytopenia secondary to hypersplenism (for which he refused splenectomy) and secondary hemochromatosis affecting the liver and heart. His serum ferritin level was markedly raised at $8325 \mathrm{ng} / \mathrm{ml}$ (NR: 13-460) and T2 weighted MRI used to assess the severity of iron loading revealed severe hepatic iron loading and dilated left and right ventricle. The 2D echocardiography showed mild biventricular dilation with normal ejection fraction suggestive of hemosiderotic cardiomyopathy. Physical examination revealed scanty axillary and pubic hair and bilateral small soft testes of $4 \mathrm{ml}$ in volume estimated by Prader
Orchidometer (Tanner stage 2). Subsequent endocrine investigations revealed panhypopituitarism (Table. 1)

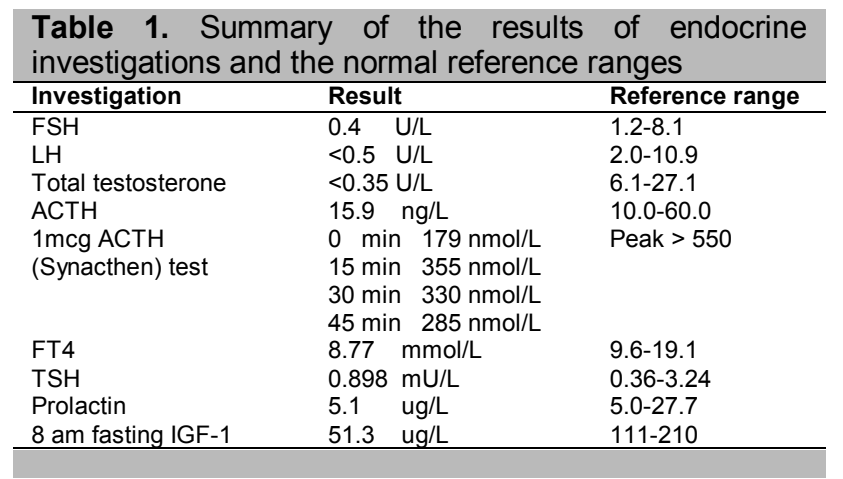

The suspicion of secondary hemochromatosis affecting the pituitary gland was confirmed with an MRI of the pituitary gland showing a diffuse hypodense pituitary consistent with iron deposition (Fig. 1). He was
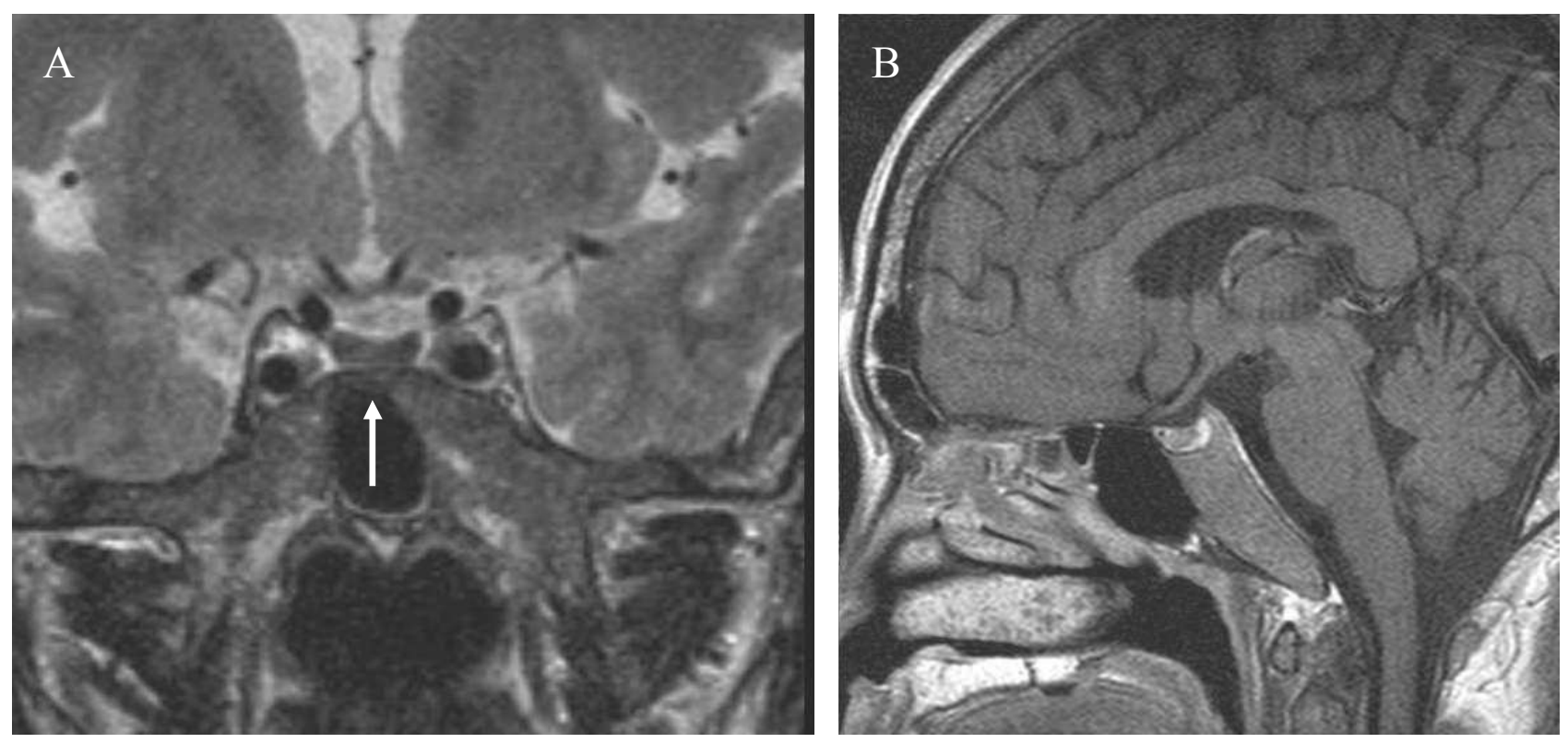

Figure 1. MRI pituitary imaging of the patient (A) Coronal T2 weighted image showing diffuse hypodense pituitary consistent with iron deposition (arrow). The gland is normal size and the stalk is central. No focal lesion was noted in the sellar or suprasellar region (B) Sagittal T1 weighted image showing normal hyperintensity of the neurohypophysis is preserved.

ISSN 0857-1074

Copyright (C) 2011 by the JAFES

Received January 26, 2011. Accepted March 26, 2011.
Corresponding author: Chng Chiaw Ling, MD

Singapore General Hospital

Outram Road, Singapore 169608

Email:chng.chiaw.ling@singhealth.com.sg 
commenced on treatment with hydrocortisone, thyroxine and testosterone replacement.

Iron deposition in patients with thalassemia can occur in a number of organs, including the heart, liver and anterior pituitary gland ${ }^{1}$. Factors that predispose to secondary hemochromatosis in thalassemia patients include chronic transfusion therapy (major cause), intestinal iron absorption triggered by chronic anemia, ineffective erythropoiesis, and as recently demonstrated, decreased serum hepcidin ${ }^{2}$. Treatment includes optimal iron chelation and hormone replacement. It is not clear at this point if the pituitary damage from secondary hemochromatosis is reversible although this has been reported in hypopituitarism related to primary hemochromatosis after aggressive iron chelation ${ }^{3}$.

\section{References}

1. Kushner, J.P., Porter, J.P. \& Olivieri, N.F. Secondary iron overload. Hematology Am Soc Hematol Educ Program, 47-61 (2001).

2. Borgna-Pignatti, C., Marsella, M. \& Zanforlin, N. The natural history of thalassemia intermedia. Ann N Y Acad Sci 1202, 214-20.

3. Gama, R., Smith, M.J., Wright, J. \& Marks, V. Hypopituitarism in primary haemochromatosis; recovery after iron depletion. Postgrad Med J 71, 297-8 (1995).

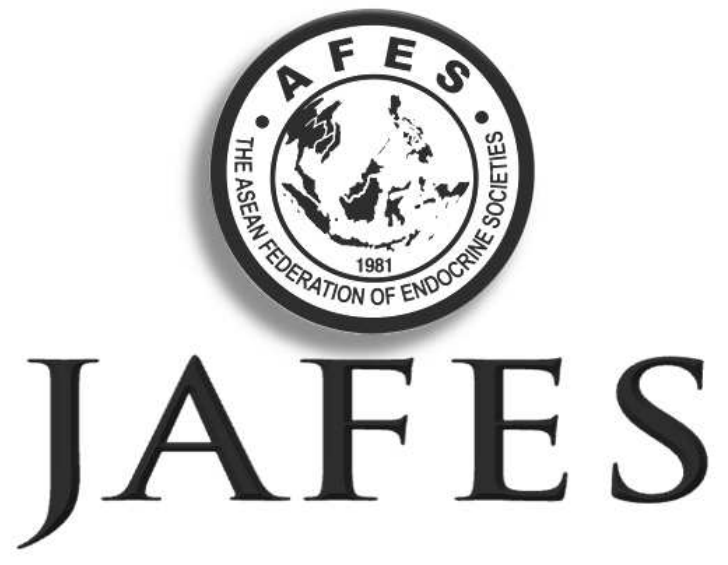

\section{Clinical controversies and disease updates are also welcome. Instructions to Authors available at www.ASEAN-endocrinejournal.org.}

